\title{
A powerful tool for browsing quick-look data in solar-terrestrial physics: "Conjunction Event Finder"
}

\author{
Yukinaga Miyashita ${ }^{1}$, Iku Shinohara ${ }^{2}$, Masaki Fujimoto ${ }^{2}$, Hiroshi Hasegawa ${ }^{2}$, \\ Keisuke Hosokawa ${ }^{3}$, Taku Takada ${ }^{4}$, and Tomoaki Hori ${ }^{1}$ \\ ${ }^{1}$ Solar-Terrestrial Environment Laboratory, Nagoya University, Nagoya, Aichi, Japan \\ ${ }^{2}$ Institute of Space and Astronautical Science, Japan Aerospace Exploration Agency, Sagamihara, Kanagawa, Japan \\ ${ }^{3}$ Department of Information and Communication Engineering, The University of Electro-Communications, Chofu, Tokyo, Japan \\ ${ }^{4}$ Kochi National College of Technology, Nankoku, Kochi, Japan
}

(Received September 13, 2010; Revised January 10, 2011; Accepted January 12, 2011; Online published February 4, 2011)

\begin{abstract}
The "Conjunction Event Finder (CEF)" is a Web tool for seamlessly browsing quick-look (QL) data from many different kinds of satellites and ground-based instruments in solar-terrestrial physics. The QL plots are generally scattered all over the world, so that browsing many of them is so far very troublesome and inefficient. Just a simple procedure on the CEF, however, generates a collection of links to the QL plots for a period of interest, allowing us to check the data much more efficiently than ever. Hence this tool is powerful in finding interesting events of conjunction observations by satellites and ground-based instruments. The CEF is available in the Data ARchives and Transmission System (DARTS) at Institute of Space and Astronautical Science (ISAS), Japan Aerospace Exploration Agency (JAXA) in Japan.
\end{abstract}

Key words: Solar-terrestrial physics, data analysis, quick-look plots, Conjunction Event Finder.

\section{Introduction}

Solar-terrestrial physics (STP) is concerned with plasma phenomena that occur on the sun and in interplanetary and near-Earth space, as well as their causal relationships and geoeffectiveness. There are many different kinds of in situ and remote observations of the sun, the solar wind, the magnetosphere, and the ionosphere by satellites and groundbased instruments. Integrated analyses of these observational data are essential in research, which is peculiar to STP.

A first step of research, particularly for case studies, is to search for interesting events and check each of them with various kinds of data. As a procedure, scientists acquire digital data of interest from the corresponding principal investigators and make the plots by themselves. Although there are a few general databases in which users can not only get digital data but also make plots, such as the Coordinated Data Analysis Web (CDAWeb) at NASA, different data are generally scattered at different institutes and universities all over the world. Different data also have different file and data formats, which have not been necessarily common in STP. Hence scientists have to acquire, read, and plot the data one by one. This is very troublesome and inefficient if many kinds of data need to be checked.

A much easier, alternative way is to browse quick-look (QL) or summary plots on the Web. Ready-made plots are often available at websites provided by principal investiga-

Copy right(c) The Society of Geomagnetism and Earth, Planetary and Space Sciences (SGEPSS); The Seismological Society of Japan; The Volcanological Society of Japan; The Geodetic Society of Japan; The Japanese Society for Planetary Sciences; TERRAPUB.

doi:10.5047/eps.2011.01.003 tors and can be quickly displayed in users' browsers one after another. Although the quality and time resolution of data are usually limited as well as the number of parameters plotted, scientists often check such useful QL plots before requesting the digital data and analyzing them in detail.

However, there are several tens or more than one hundred of websites for QL plots at different institutes and universities all over the world. Scientists, therefore, have to open a new window and input a date and period as well as a data type of interest for each plot. This procedure is still very troublesome and inefficient if many plots, even several, need to be checked in studies with multisatellite and ground-based observations. Thus we desire to browse QL plots more seamlessly.

\section{Conjunction Event Finder}

As a solution for mitigating the inefficiency of browsing QL plots, we have developed the Conjunction Event Finder (CEF), which collectively generates a large number of links to QL plots of various satellite and groundbased measurements for a period of interest. This tool is available in the Data ARchives and Transmission System (DARTS) at Institute of Space and Astronautical Science (ISAS), Japan Aerospace Exploration Agency (JAXA) in Japan (http://darts.isas.jaxa.jp/stp/cef/cef.cgi). As shown in Fig. 1, once selecting a two-hour period of interest and clicking the "Submit" button on the CEF main page, the links to various QL plots for the period will be collectively generated. The data include those from satellites observing the sun, the solar wind, the magnetosphere, and the ionosphere, those from ground-based instruments, such as radars, all-sky imagers, and magnetometers, and those of 
CEF Main Page: http://darts.isas.jaxa.jp/stp/cef/cef.cgi

(1) Select a period of interest, and the links to QL plots for the period will be generated.

\section{Conjunction Event Finder for 1992-2012}

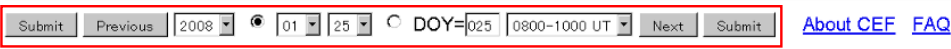
Orbit | GT I CL I DSP I THM | Sun I SW I Magnetosphere | Low-Altitude | Radar | Aurora | Geomag | Others - Plots for the above period o Related Sites

(2) Open various QL plots.

Orbit

Spacecraft Footprints and Ground-Based Instruments: Polar 2 hours- Polar 1 day - Mid-Latitude 2 hours Mid-Latitude 1 day (ISAS/JAXA)

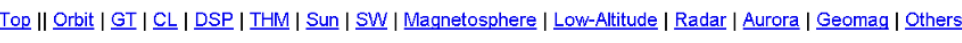
Geotail

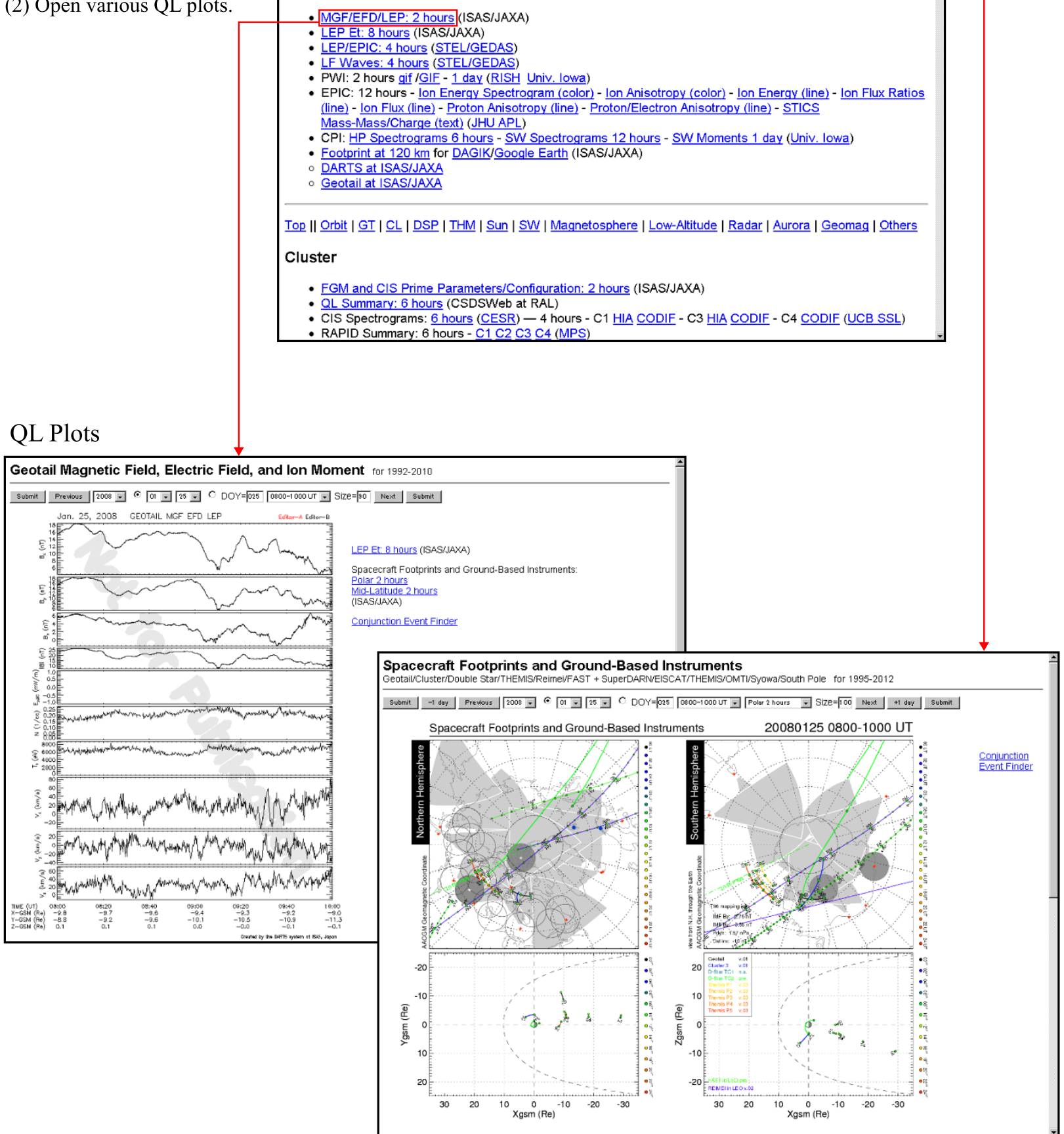

Fig. 1. Illustration of how to use the Conjunction Event Finder (CEF) main page and examples of the QL plots available in DARTS at ISAS, JAXA. 
satellite orbits and their ionospheric footprints. (The first version was released at the beginning of September 2006. As of September 2010, there are more than 70 links to QL plots.) This very simple procedure allows us to seamlessly browse a number of QL plots scattered all over the world and check the data much more efficiently than ever. So far scientists had to open a new window to visit each website and input a date and period of interest, instead of visiting only one website such as the CEF.

The CEF main page looks simple and does not necessarily use special and up-to-date techniques. The CEF main page is dynamically generated by a common gateway interface (CGI) written in Perl to make a collection of links to QL plots for a period of interest. There are two methods for making the direct link to a QL plot. Most websites in the world use CGIs in their servers to display QL plots. A selected date, period, data type, and other parameters (if necessary) are provided to their CGIs as arguments. If possible, the CEF specifies the URLs of the CGIs, explicitly including the arguments, to provide them to the CGIs and display the QL plots of interest immediately. For example, in the case of the Geotail QL plots that are available in DARTS (see Fig. 1), if the necessary arguments turn out to be the values of $Y Y Y Y$ (year), $M M$ (month), $D D$ (day), $P P P P$ (period), and SSS (plot size), and be given to the CGI as http://darts.isas.jaxa.jp/stp/cef/geotail/geotail.cgi?year= $Y Y Y Y$ \& month $=Y Y \&$ day $=D D \&$ period $=P P P P \&$ size $=S S S \&$ jump=Submit to display the QL plot of interest, then the CEF can easily generate the direct link of the QL plot. In some websites, the above method is not allowed or CGIs are not used, but the image files of QL plots are available. In this case, the direct links to the files are generated by the CGI of the CEF. Here it is necessary that the image files be named with simple rules. For example, the URL of the Geotail QL plot is given as http://darts.isas.jaxa.jp/stp/cef/geotail/2hours/ mgflepgif/YYYY/YYMMDD_PPPP_gtlmgflep.gif, where $Y Y$ is the last two digits of $Y Y Y Y$. Thus, in spite of the simple idea and technique, the CEF main page is a very powerful tool for browsing QL plots.

In addition to the CEF main page, we have made QL plots of the data from the Geotail (Nishida, 1994), Cluster (Escoubet et al., 1997, 2001), Double Star (TC1 and TC2) (Liu et al., 2005), and THEMIS (Angelopoulos, 2008) satellites, which are also available in DARTS at ISAS, JAXA. Furthermore, we made plots of definitive and predictive orbits and ionospheric footprints of the Geotail, Cluster, Double Star, THEMIS, Reimei (Saito et al., 2001), and FAST (Carlson et al., 1998) satellites, along with the locations and/or the fields of view of the ground-based instruments of the SuperDARN (Greenwald et al., 1995) and EISCAT (Rishbeth and Williams, 1985) radars, and the THEMIS (Mende et al., 2008), OMTI (Shiokawa et al., 1999, 2009b), and Syowa and South Pole Station (Ejiri et al., 1999) all-sky imagers. The links to these QL plots can be found at the CEF main page and also http://darts.isas.jaxa.jp/stp/.

The collection of links to QL plots and the QL plots of the CEF in DARTS are very helpful in studying conjunction events using multisatellite and ground-based mea- surements, that is, in finding interesting events in the data of a satellite (e.g., Hasegawa et al., 2008 for a quasi-continuous tailward-of-the-cusp reconnection event observed by Cluster) and in checking the locations of other satellites, the conjunction with ground-based instruments, and their observational data for selected events (e.g., Takada et al., 2008 for local field-aligned currents in the magnetotail and the ionosphere from conjunction observations by Cluster, Double Star, MIRACLE ground magnetometers, and the auroral camera onboard the IMAGE satellite; and Miyashita et al., 2008 for substorm-associated convection enhancement from conjunction observations by Geotail, SuperDARN, and other satellites and ground-based instruments). In addition to past observations, the plots of predictive orbits and ionospheric footprints of the satellites help us to plan future campaign observations in satellite-ground conjunction (e.g., Shiokawa et al., 2009a for substorm auroral initial brightenings from their campaign observations by their own high time resolution all-sky cameras in conjunction with THEMIS all-sky cameras and satellites).

In conclusion, separate QL plots available at different websites generally may not be necessarily user-friendly. Even a simple tool such as the CEF, however, will mitigate the inefficiency, allowing us to seamlessly browse various QL plots of multisatellite and ground-based measurements and find interesting conjunction events much more efficiently than ever.

Acknowledgments. The CEF was developed when Y. M. worked at ISAS, JAXA in 2006 through 2008. We thank those who provided us with the data and/or information used for the QL plots available in DARTS at ISAS, JAXA: T. Mukai, Y. Saito, S. Kokubun, T. Nagai, K. Tsuruda, and H. Hayakawa for Geotail; C. P. Escoubet, E. A. Lucek, H. Rème, and European Space Agency (ESA) for Cluster; C. P. Escoubet, Z. X. Liu, R. Nakamura, C. M. Carr, H. Rème, and ESA for Double Star; V. Angelopoulos, K. H. Glassmeier, U. Auster, W. Baumjohann, C. W. Carlson, J. P. McFadden, D. Larson, R. P. Lin, S. Mende, Space Sciences Laboratory, University of California, Berkeley (SSL/UCB), and NASA SSCWeb for THEMIS; M. Hirahara and K. Asamura for Reimei; C. W. Carlson, K. Seki, and SSL/UCB for FAST; NASA OMNIWeb for OMNI 2; Johns Hopkins University Applied Physics Laboratory for SuperDARN; Suomen Akatemia in Finland, Centre National de la Recherche Scientifique in France, Max-Planck-Gesellschaft in Germany, National Institute of Polar Research (NIPR) in Japan, Norges forskningsrad in Norway, Vetenskapsradet in Sweden, and Particle Physics and Astronomy Research Council in United Kingdom for EISCAT; K. Shiokawa for OMTI; and NIPR for the Syowa and South Pole Stations. We thank Y. Kasaba for his useful comments.

\section{References}

Angelopoulos, V., The THEMIS mission, Space Sci. Rev., 141, 5-34, doi:10.1007/s11214-008-9336-1, 2008.

Carlson, C. W., R. F. Pfaff, and J. G. Watzin, The Fast Auroral SnapshoT (FAST) mission, Geophys. Res. Lett., 25, 2013-2016, doi:10.1029/98GL01592, 1998.

Ejiri, M., T. Aso, M. Okada, M. Tsutsumi, M. Taguchi, N. Sato, and S. Okano, Japanese research project on Arctic and Antarctic observations of the middle atmosphere, Adv. Space Res., 24, 1689-1692, doi:10.1016/S0273-1177(99)00335-X, 1999.

Escoubet, C. P., R. Schmidt, and M. L. Goldstein, ClusterScience and mission overview, Space Sci. Rev., 79, 11-32, doi:10. 1023/A:1004923124586, 1997.

Escoubet, C. P., M. Fehringer, and M. Goldstein, The Cluster mission, Ann. Geophys., 19, 1197-1200, doi:10.5194/angeo-19-1197-2001, 2001.

Greenwald, R. A. et al., DARN/SuperDARN: A global view of the 
dynamics of high-latitude convection, Space Sci. Rev., 71, 761-796, doi:10.1007/BF00751350, 1995.

Hasegawa, H., A. Retinò, A. Vaivads, Y. Khotyaintsev, R. Nakamura, T. Takada, Y. Miyashita, H. Rème, and E. A. Lucek, Retreat and reformation of X-line during quasi-continuous tailward-of-the-cusp reconnection under northward IMF, Geophys. Res. Lett., 35, L15104, doi:10.1029/2008GL034767, 2008.

Liu, Z. X., C. P. Escoubet, Z. Pu, H. Laakso, J. K. Shi, C. Shen, and M. Hapgood, The Double Star mission, Ann. Geophys., 23, 2707-2712, doi:10.5194/angeo-23-2707-2005, 2005.

Mende, S. B., S. E. Harris, H. U. Frey, V. Angelopoulos, C. T. Russell, E. Donovan, B. Jackel, M. Greffen, and L. M. Peticolas, The THEMIS array of ground-based observatories for the study of auroral substorms, Space Sci. Rev., 141, 357-387, doi:10.1007/s11214-008-9380-x, 2008.

Miyashita, Y. et al., Response of large-scale ionospheric convection to substorm expansion onsets: A case study, J. Geophys. Res., 113, A12309, doi:10.1029/2008JA013586, 2008.

Nishida, A., The Geotail mission, Geophys. Res. Lett., 21, 2871-2873, doi:10.1029/94GL01223, 1994.

Rishbeth, H. and P. J. S. Williams, The EISCAT ionospheric radar: The system and its early results, Q.J.R. Astron. Soc., 26, 478-512, 1985.
Saito, H. et al., INDEX: Piggy-back satellite for aurora observation and technology demonstration, Acta Astronaut., 48, 723-735, doi:10.1016/S0094-5765(01)00079-0, 2001.

Shiokawa, K., Y. Katoh, M. Satoh, M. K. Ejiri, T. Ogawa, T. Nakamura, T. Tsuda, and R. H. Wiens, Development of Optical Mesosphere Thermosphere Imagers (OMTI), Earth Planets Space, 51, 887-896, 1999.

Shiokawa, K. et al., Longitudinal development of a substorm brightening arc, Ann. Geophys., 27, 1935-1940, doi:10.5194/angeo-27-1935-2009, 2009a.

Shiokawa, K., Y. Otsuka, and T. Ogawa, Propagation characteristics of nighttime mesospheric and thermospheric waves observed by optical mesosphere thermosphere imagers at middle and low latitudes, Earth Planets Space, 61, 479-491, 2009b.

Takada, T. et al., Local field-aligned currents in the magnetotail and ionosphere as observed by a Cluster, Double Star, and MIRACLE conjunction, J. Geophys. Res., 113, A07S20, doi:10.1029/2007JA012759, 2008.

Y. Miyashita (e-mail: miyasita@stelab.nagoya-u.ac.jp), I. Shinohara, M. Fujimoto, H. Hasegawa, K. Hosokawa, T. Takada, and T. Hori 